\title{
Interaction correction to the conductance of a ballistic conductor
}

\author{
Piet W. Brouwer and Joern N. Kupferschmidt \\ Laboratory of Atomic and Solid State Physics, Cornell University, Ithaca, NY 14853, USA and \\ Arnold Sommerfeld Center for Theoretical Physics, \\ Ludwig-Maximilians-Universität, 80333 München, Germany
}

(Dated: October 29, 2018)

\begin{abstract}
In disordered metals, electron-electron interactions are the origin of a small correction to the conductivity, the "Altshuler-Aronov correction". Here we investigate the Altshuler-Aronov correction $\delta G_{\mathrm{AA}}$ of a conductor in which the electron motion is ballistic and chaotic. We consider the case of a double quantum dot, which is the simplest example of a ballistic conductor in which $\delta G_{\mathrm{AA}}$ is nonzero. The fact that the electron motion is ballistic leads to an exponential suppression of $\delta G_{\mathrm{AA}}$ if the Ehrenfest time is larger than the mean dwell time $\tau_{\mathrm{D}}$ or the inverse temperature $\hbar / T$.
\end{abstract}

PACS numbers: 73.23.-b, 05.45.Mt, 73.20.Fz

There are two quantum corrections of comparable magnitude to the conductivity of a disordered normal metal at low temperatures: the weak localization correction, which has its origin in the constructive interference of electrons traveling along time-reversed paths [1, 2], and the "Altshuler-Aronov correction", which is caused by electron-electron interactions [3]. These two corrections can be distinguished through their different dependences on temperature and magnetic field. For mesoscopic conductors, which are characterized by their conductance, not their conductivity, weak localization and the Altshuler-Aronov correction represent small changes to the mean conductance after taking an average over different disorder configurations [4].

Although weak localization was discovered in the context of disordered metals, it also occurs if the electron motion is ballistic and the only source of scattering is specular reflection off sample boundaries or artificial macroscopic scattering sites [5, 6, 7]. There is an important difference between the two cases, however: For weak localization in ballistic conductors a key role is played by the Ehrenfest time $\tau_{\mathrm{E}}$ [8], a time which has no counterpart in disordered metals. The Ehrenfest time separates regimes of classical-deterministic and quantum-probabilistic motion [9, 10], thus serving as a short-time "threshold" for quantum corrections in a ballistic conductor. The weak localization correction $\delta G_{\mathrm{WL}}$ has an exponential dependence on $\tau_{\mathrm{E}}$ if $\tau_{\mathrm{E}}$ is large, $\delta G_{\mathrm{WL}} \propto \exp \left(-\tau_{\mathrm{E}} / \tau_{\mathrm{D}}-\tau_{\mathrm{E}} / \tau_{\phi}\right)$, where $\tau_{\mathrm{D}}$ is the dwell time and $\tau_{\phi}$ the dephasing time [8, 11, 12].

In this letter we show that $\tau_{\mathrm{E}}$ also serves as a shorttime threshold for the Altshuler-Aronov correction $\delta G_{\mathrm{AA}}$ in a ballistic conductor, in a manner quite similar to the way it appears in the theory of weak localization and other quantum corrections that do not rely on electronelectron interactions. In particular, we show that $\delta G_{\mathrm{AA}}$ has an exponential dependence on $\tau_{\mathrm{E}}$ if $\tau_{\mathrm{E}}$ is large,

$$
\delta G_{\mathrm{AA}} \propto e^{-\tau_{\mathrm{E}} / \tau_{\mathrm{D}}-2 \pi T \tau_{\mathrm{E}} / \hbar},
$$

where $T$ is the temperature. The exponential sensitivity

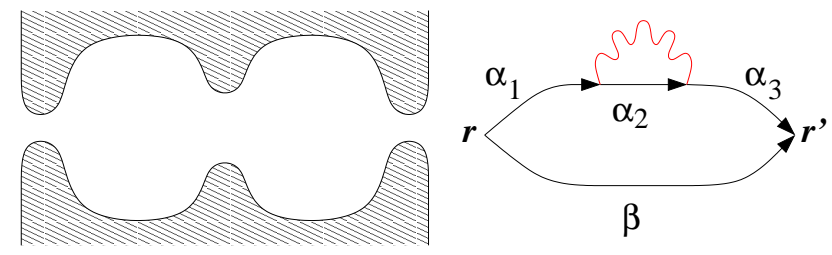

FIG. 1: (Color online) Schematic drawing of a double quantum dot (left), with a generic set of four trajectories that contributes to the interaction correction to the conductance (right). The wiggly line represents the interaction propagator.

to temperature is special to the interaction correction; Although weak localization depends on $T$ implicitly via the temperature-dependence of the dephasing time, the corresponding exponential dependence is much weaker since $\tau_{\phi} \gg \hbar / T[4]$.

For most studies of quantum corrections in ballistic conductors, the geometry of choice is a ballistic cavity or "quantum dot". This geometry is not suitable for a theory of the Altshuler-Aronov correction, however, because $\delta G_{\mathrm{AA}}=0$ in a ballistic quantum dot [13, 14]. Therefore, we here calculate $\delta G_{\mathrm{AA}}$ for a "double quantum dot", a device consisting of two quantum dots coupled by a ballistic contact. Although $\delta G_{\mathrm{AA}}$ will be quantitatively different in other geometries, we believe that the qualitative features mentioned above carry over to the general case.

A schematic drawing of a double quantum dot is shown in Fig. 1. The two quantum dots are connected to source and drain reservoirs via ballistic contacts of conductances $G_{1}, G_{2} \gg e^{2} / h$ respectively; They are connected with each other via a ballistic contact with conductance $G_{\mathrm{c}}$. The classical electron dynamics in each quantum dot is ballistic and chaotic, with Lyapunov exponent $\lambda$. (We assume the same Lyapunov exponent for both quantum dots.) The Ehrenfest time then reads

$$
\tau_{\mathrm{E}}=\lambda^{-1} \ln \left(k_{F} L\right),
$$

where $k_{F}$ is the Fermi wavenumber and $L$ the dot size. We take the semiclassical limit $k_{F} L \gg 1$, so that the log- 
arithm in Eq. (2) is large. For a ballistic double quantum dot, the disorder average is replaced by an average over the Fermi energy or over variations of the dot's shape.

In the semiclassical limit the electron-electron interaction in the double quantum dot takes a particularly simple form: It is determined by the capacitances $C_{1,2}$ of the two dots and their mutual capacitance $C_{\mathrm{c}}$ only. (For the case of a single quantum dot the corresponding interaction is known as the "universal interaction Hamiltonian" [15].) As a result, the interaction propagator $\mathcal{D}\left(\mathbf{r}_{1}, \mathbf{r}_{2} ; \omega\right)$ for the double dot is spatially homogeneous inside each dot, so that it may be represented by a $2 \times 2$ matrix $\tilde{\mathcal{D}}$, where the matrix indices refer to the two quantum dots,

$$
\tilde{\mathcal{D}}^{\mathrm{R}}(\omega)^{-1}=-\tilde{C} / e^{2}-\left[\tilde{\nu}^{-1}-i e^{2} \omega(\hbar \tilde{G})^{-1}\right]^{-1}
$$

with $\tilde{C}_{m n}=C_{m} \delta_{m n}+C_{\mathrm{c}}(-1)^{m+n}, \tilde{G}_{m n}=G_{m} \delta_{m n}+$ $G_{\mathrm{c}}(-1)^{m+n}$, and $\tilde{\nu}_{m n}=\nu_{m} \delta_{m n}$, where $\nu_{1}$ and $\nu_{2}$ are the level densities in each dot. For all frequencies of interest one may neglect the first term in Eq. (3) and approximate $\tilde{\mathcal{D}}^{\mathrm{R}}(\omega)=i e^{2} \omega(\hbar \tilde{G})^{-1}-\tilde{\nu}^{-1}$.
The Altshuler-Aronov correction $\delta G_{\mathrm{AA}}$ can be calculated from the interaction correction to the singleelectron Green function $\mathcal{G}\left(\mathbf{r}, \mathbf{r}^{\prime} ; \omega\right)$ (before the ensemble average). Without interactions, the relation between $\mathcal{G}$ and the conductance $G$ is given by the Kubo formula,

$$
G=\frac{e^{2} \hbar}{\pi} \hat{v}_{x} \hat{v}_{x^{\prime}} \int d y d y^{\prime} \int d \xi \frac{\mathcal{G}^{\mathrm{R}}\left(\mathbf{r}, \mathbf{r}^{\prime} ; \xi\right) \mathcal{G}^{\mathrm{A}}\left(\mathbf{r}^{\prime}, \mathbf{r} ; \xi\right)}{4 T \cosh ^{2}(\xi / 2 T)}
$$

where $x$ and $x^{\prime}\left(y\right.$ and $\left.y^{\prime}\right)$ are longitudinal (transverse) coordinates in the source and drain contacts, respectively, and

$$
\begin{aligned}
\hat{v}_{x} \mathcal{G}(\mathbf{r}, \cdot) \mathcal{G}(\cdot, \mathbf{r})= & \frac{\hbar e}{2 m i}\left[\left(\partial_{x} \mathcal{G}(\mathbf{r}, \cdot)\right) \mathcal{G}(\cdot, \mathbf{r})\right. \\
& \left.-\mathcal{G}(\mathbf{r}, \cdot) \partial_{x} \mathcal{G}(\cdot, \mathbf{r})\right] .
\end{aligned}
$$

The Altshuler-Aronov correction $\delta G_{\mathrm{AA}}$ then follows from Eq. (4) if one makes the substitution $\mathcal{G}^{\mathrm{R}, \mathrm{A}} \rightarrow \mathcal{G}^{\mathrm{R}, \mathrm{A}}+$ $\delta \mathcal{G}^{\mathrm{R}, \mathrm{A}}$, with $[3,16$ ]

$$
\begin{aligned}
\delta \mathcal{G}^{\mathrm{R}}\left(\mathbf{r}, \mathbf{r}^{\prime} ; \xi\right)= & \int \frac{d \omega}{4 \pi i} \tanh \left(\frac{\omega-\xi}{2 T}\right) \int d \mathbf{r}_{1} d \mathbf{r}_{2} \mathcal{G}^{\mathrm{R}}\left(\mathbf{r}, \mathbf{r}_{1} ; \xi\right) \mathcal{G}^{\mathrm{R}}\left(\mathbf{r}_{2}, \mathbf{r}^{\prime} ; \xi\right) \\
& \times\left\{\mathcal{D}^{\mathrm{R}}\left(\mathbf{r}_{1}, \mathbf{r}_{2} ; \omega\right) \mathcal{G}^{\mathrm{A}}\left(\mathbf{r}_{1}, \mathbf{r}_{2} ; \xi-\omega\right)-\mathcal{D}^{\mathrm{A}}\left(\mathbf{r}_{1}, \mathbf{r}_{2} ; \omega\right) \mathcal{G}^{\mathrm{R}}\left(\mathbf{r}_{1}, \mathbf{r}_{2} ; \xi-\omega\right)\right\}
\end{aligned}
$$

and a similar expression for $\delta \mathcal{G}^{\mathrm{A}}$. Notice that Eq. (5) represents the Fock contribution to $\delta \mathcal{G}$ only. The Hartree contribution to $\delta \mathcal{G}$ vanishes because of the special form of the interaction $\mathcal{D}\left(\mathbf{r}_{1}, \mathbf{r}_{2} ; \omega\right)$, see Eq. (3) above.

Together, Eqs. (3)- (5) express $\delta G_{\mathrm{AA}}$ as an integral over a product of four single electron Green functions. What remains to be done is to calculate the ensemble average. Hereto, we use a semiclassical approach inspired by the semiclassical calculation of the weak localization correction to the conductance in Ref. [17]. First, we use the standard expression of the Green function $\mathcal{G}\left(\mathbf{r}, \mathbf{r}^{\prime} ; \xi\right)$ as a sum over classical trajectories $\alpha$ connecting the points $\mathbf{r}$ and $\mathbf{r}^{\prime}[18]$,

$$
\begin{aligned}
\mathcal{G}^{\mathrm{R}}\left(\mathbf{r}, \mathbf{r}^{\prime} ; \xi\right) & =\mathcal{G}^{\mathrm{A}}\left(\mathbf{r}^{\prime}, \mathbf{r} ; \xi\right)^{*} \\
& =\frac{2 \pi}{(2 \pi i \hbar)^{3 / 2}} \sum_{\alpha} A_{\alpha} e^{i \mathcal{S}_{\alpha}(\xi) / \hbar}
\end{aligned}
$$

where $A_{\alpha}$ is the stability amplitude of $\alpha$ and $\mathcal{S}_{\alpha}$ its classical action. Actions at different energies are related via

$$
\mathcal{S}_{\alpha}(\xi)-\mathcal{S}_{\alpha}(\xi-\omega)=\omega \tau_{\alpha},
$$

where $\tau_{\alpha}$ is travel time from $\mathbf{r}^{\prime}$ to $\mathbf{r}$ along $\alpha$. Substitution of Eq. (6) into Eqs. (4) and (5) expresses $\delta G_{\mathrm{AA}}$ as a sum over four classical trajectories $\alpha_{1}, \alpha_{2}, \alpha_{3}$, and $\beta$ [Fig.
1. right panel]. For each set of four classical trajectories there are four contributions to $\delta G_{\mathrm{AA}}$, corresponding to the two terms in Eq. (5) and the two terms in the corresponding expression for $\delta \mathcal{G}^{\mathrm{A}}$. The four cases differ by the assignment which of the trajectories $\alpha_{1}, \alpha_{2}, \alpha_{3}$, and $\beta$ correspond to retarded and which correspond to advanced Green functions. Each configuration of trajectories contributes to the average conductance $\langle G\rangle$ only if the total action difference $\Delta \mathcal{S}$ is of order $\hbar$ systematically. This occurs only if the 'retarded' and 'advanced' trajectories are piecewise equal, up to classical phase space distances of order $\hbar^{1 / 2}$ and below [8, 17]. Five possible configurations of classical trajectories that meet this requirement are shown in Fig. 2. In Fig. 2a, $\alpha_{1}, \alpha_{2}$, and $\alpha_{3}$ represent retarded Green functions, whereas $\beta$ represents an advanced Green function. In Fig. $2 \mathrm{~b}-\mathrm{e}, \alpha_{1}$ and $\alpha_{3}$ represent retarded Green functions, whereas $\alpha_{2}$ and $\beta$ represent advanced Green functions. There are five more trajectory configurations that contribute to $\delta G_{\mathrm{AA}}$ which can be obtained from those of Fig. 2 by interchanging the roles of advanced and retarded Green functions.

In Fig. 2 a, the points $\mathbf{r}_{1}$ and $\mathbf{r}_{2}$ are on the trajectory $\beta$ (up to a quantum uncertainty), and the three trajectories $\alpha_{1}, \alpha_{2}$, and $\alpha_{3}$ are equal to different successive segments of $\beta$ (again up to quantum uncertainties). In Figs. 2b-e, 


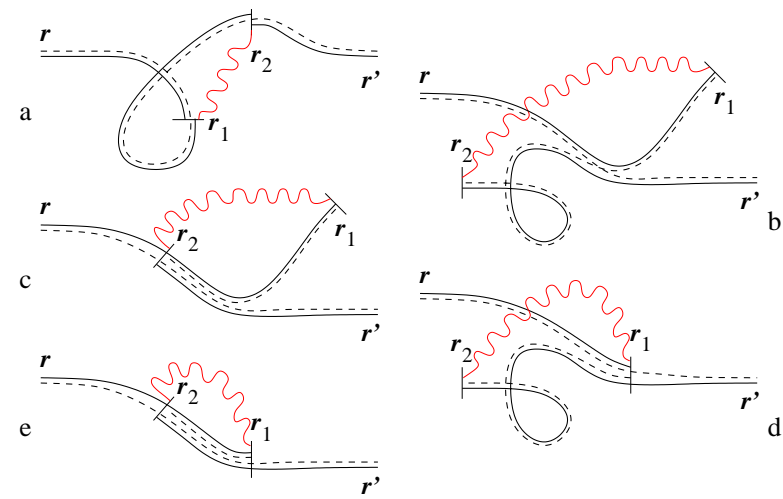

FIG. 2: (Color online) Schematic drawing of five configurations of classical trajectories contributing to $\delta G_{\mathrm{AA}}$. Solid (dashed) trajectories represent retarded (advanced) Green functions. Five more trajectory configurations are obtained by interchanging the roles of retarded and advanced Green functions. The wiggly lines represent the advanced interaction propagator.

the situation is more complicated because the two points $\mathbf{r}_{1}$ and $\mathbf{r}_{2}$ need not be on $\beta$ for this contribution to $\delta G_{\mathrm{AA}}$. If both $\mathbf{r}_{1}$ and $\mathbf{r}_{2}$ are not on $\beta$, as in Fig. $2 \mathrm{~b}$, the trajecto- ries $\beta$ and $\alpha_{2}$ undergo a "small angle encounter". Small angle encounters play a crucial role in the theory of weak localization and the shot noise power in ballistic conductors [8, 17, 19, 20, 21, 22]. The condition that the action difference $\Delta \mathcal{S}=S_{\alpha_{1}}-\mathcal{S}_{\alpha_{2}}+\mathcal{S}_{\alpha_{3}}-\mathcal{S}_{\beta}$ be of order $\hbar$ translates to the condition that the duration of the encounter be equal to the Ehrenfest time $\tau_{\mathrm{E}}$ 8, 17]. The same is true if one of the points $\mathbf{r}_{1}$ and $\mathbf{r}_{2}$ is on $\beta$ and one is not, as in Fig. 2c and d. If both points $\mathbf{r}_{1}$ and $\mathbf{r}_{2}$ are on $\beta$, as in Fig. 2e, the travel time between $\mathbf{r}_{1}$ and $\mathbf{r}_{2}$ must be less than $\tau_{\mathrm{E}}[20]$.

The configurations of Figs. 2a and e are essentially one dimensional. Their contributions are found to cancel precisely if the travel time $\tau_{\alpha_{2}}$ between $\mathbf{r}_{1}$ and $\mathbf{r}_{2}$ is less than $\tau_{\mathrm{E}}$. The configurations of Figs. 2 $\mathrm{b}-\mathrm{d}$ require a summation over trajectories involved in a small angle encounter, taking into account the proper action difference $\Delta \mathcal{S}$. For the present case, where the interaction propagator $\mathcal{D}\left(\mathbf{r}_{1}, \mathbf{r}_{2} ; \omega\right)$ is independent of the precise location of the coordinates $\mathbf{r}_{1}$ and $\mathbf{r}_{2}$ inside each quantum dot, this summation is essentially identical to that needed for the calculation of the shot noise power. Following the method of Refs. [20, 21, 22], one then finds

$$
\begin{aligned}
\delta G_{\mathrm{AA}}=\frac{e^{2}}{h} & \int \frac{d \omega}{i \hbar} \mathcal{F}(\omega / T) \sum_{m, n} \tilde{\mathcal{D}}_{m n}^{\mathrm{A}}(\omega) \nu_{n}\left\{\int_{\tau_{\mathrm{E}}}^{\infty} d t P_{m}^{\mathrm{L}} P_{m n}(t) P_{n}^{\mathrm{R}} e^{-i \omega t / \hbar}\right. \\
& \left.-\sum_{k, l} \int_{0}^{\infty} d t_{2} d t_{1}\left[\partial_{\tau_{\mathrm{E}}}+\delta\left(t_{1}\right)+\delta\left(t_{2}\right)\right] P_{m k}\left(t_{2}\right) P_{k}^{\mathrm{L}} P_{k l}\left(\tau_{\mathrm{E}}\right) P_{l}^{\mathrm{R}} P_{l n}\left(t_{1}\right) e^{-i \omega\left(\tau_{\mathrm{E}}+t_{1}+t_{2}\right) / \hbar}\right\}+ \text { c.c. },
\end{aligned}
$$

where $\mathcal{F}(x)=(\sinh x-x) /(\cosh x-1), P_{n}^{\mathrm{L}}$ and $P_{n}^{\mathrm{R}}=1-P_{n}^{\mathrm{L}}$ are the classical probabilities that an electron in dot $n$ exits the system through the left or right contacts, respectively, and $P_{m n}(t)$ is the classical probability that an electron in $\operatorname{dot} n$ is in dot $m$ after a time $t$. The first term in Eq. (8) comes from trajectory configurations of Fig. $2 \mathrm{a}$ and e; the second term comes from Fig. $2 \mathrm{~b}-\mathrm{d}$. In matrix language, one has $P_{k l}(t)=\left[\exp \left(-\tilde{G} t / e^{2} \tilde{\nu}\right)\right]_{k l}$, where the $2 \times 2$ matrices $\tilde{G}$ and $\tilde{\nu}$ were defined below Eq. (3).

In the special case of a symmetric double quantum dot with $G_{1}=G_{2} \equiv G_{\mathrm{d}}$ and $\nu_{1}=\nu_{2} \equiv \nu$, and neglecting the first term in Eq. (3), Eq. (8) simplifies to

$$
\delta G_{\mathrm{AA}}=\frac{e^{2}}{\pi \hbar} \frac{G_{\mathrm{d}} G_{\mathrm{c}}^{2}\left(\tau_{\mathrm{D}-} e^{-\tau_{\mathrm{E}} / \tau_{\mathrm{D}+}}+\tau_{\mathrm{D}+} e^{-\tau_{\mathrm{E}} / \tau_{\mathrm{D}-}}\right)}{\left(G_{\mathrm{d}}+2 G_{\mathrm{c}}\right)^{3}} \operatorname{Im} \int d \omega \frac{e^{-i \omega \tau_{\mathrm{E}}} \mathcal{F}(\hbar \omega / T)}{\left(1+i \omega \tau_{\mathrm{D}+}\right)\left(1+i \omega \tau_{\mathrm{D}-}\right)},
$$

where $\tau_{\mathrm{D}+}=e^{2} \nu / G_{\mathrm{d}}$ and $\tau_{\mathrm{D}-}=e^{2} \nu /\left(G_{\mathrm{d}}+2 G_{\mathrm{c}}\right)$ are the characteristic dwell times of the double quantum dot. The indices $+(-)$ refer to relaxation of (anti)symmetric charge configurations. The frequency integral in Eq. (9) can not be performed in closed form, except in asymptotic limits. If $\tau_{\mathrm{E}} \ll \min \left(\tau_{\mathrm{D} \pm}, \hbar / T\right)$, one recovers the result of random matrix theory [23],

$$
\delta G_{\mathrm{AA}}=-\frac{2 e^{2}}{\pi \hbar} \frac{G_{\mathrm{d}} G_{\mathrm{c}}^{2}}{\left(G_{\mathrm{d}}+2 G_{\mathrm{c}}\right)^{3}} \frac{\tau_{\mathrm{D}+}+\tau_{\mathrm{D}-}}{\tau_{\mathrm{D}+}-\tau_{\mathrm{D}-}} \ln \frac{\tau_{\mathrm{D}+}}{\tau_{\mathrm{D}-}}
$$

if $\tau_{\mathrm{D}} T \ll \hbar$, and

$$
\delta G_{\mathrm{AA}}=-\frac{e^{2}}{3 T} \frac{G_{\mathrm{d}} G_{\mathrm{c}}^{2}}{\left(G_{\mathrm{d}}+2 G_{\mathrm{c}}\right)^{3}} \frac{\tau_{\mathrm{D}+}+\tau_{\mathrm{D}-}}{\tau_{\mathrm{D}+} \tau_{\mathrm{D}-}}
$$

if $\tau_{\mathrm{D}} T \gg \hbar$. As soon as $\tau_{\mathrm{E}}$ becomes comparable to $\tau_{\mathrm{D}}$ or $\hbar / 2 \pi T, \delta G_{\mathrm{AA}}$ acquires a dependence on $\tau_{\mathrm{E}}$, which becomes exponential in the limit of large Ehrenfest times,

$$
\delta G_{\mathrm{AA}}=-\frac{2 e^{2}}{\pi \hbar \tau_{\mathrm{E}}} \frac{G_{\mathrm{d}} G_{\mathrm{c}}^{2}\left(\tau_{\mathrm{D}-} e^{-\tau_{\mathrm{E}} / \tau_{\mathrm{D}+}}+\tau_{\mathrm{D}+} e^{-\tau_{\mathrm{E}} / \tau_{\mathrm{D}-}}\right)}{\left(G_{\mathrm{d}}+2 G_{\mathrm{c}}\right)^{3}}
$$


if $\tau_{\mathrm{D}} \ll \tau_{\mathrm{E}} \ll \hbar / T$, and

$$
\begin{aligned}
\delta G_{\mathrm{AA}}= & -\frac{2 e^{2}}{\pi \hbar} \frac{G_{\mathrm{d}} G_{\mathrm{c}}^{2} \tau_{\mathrm{E}} e^{-2 \pi T \tau_{\mathrm{E}} / \hbar}}{\left(G_{\mathrm{d}}+2 G_{\mathrm{C}}\right)^{3}} \\
& \times \frac{\tau_{\mathrm{D}-} e^{-\tau_{\mathrm{E}} / \tau_{\mathrm{D}+}}+\tau_{\mathrm{D}+} e^{-\tau_{\mathrm{E}} / \tau_{\mathrm{D}-}}}{\left(\tau_{\mathrm{D}+}+\hbar / 2 \pi T\right)\left(\tau_{\mathrm{D}-}+\hbar / 2 \pi T\right)}
\end{aligned}
$$

if $\tau_{\mathrm{E}} \gg \hbar / T$. Equations (12) and (13) reproduce the general exponential dependence of Eq. (1), with $\tau_{\mathrm{D}}=$ $\tau_{\mathrm{D}}+$ taken to be the larger of the two characteristic dwell times. For a generic (non-symmetric) double quantum dot one finds the same dependence on $\tau_{\mathrm{E}}$ from the general expression of Eq. (8).

Let us now compare the interaction correction $\delta G_{\mathrm{AA}}$ to the weak localization correction $\delta G_{\mathrm{WL}}$ in the same system, for which one finds [22]

$$
\delta G_{\mathrm{WL}}=\frac{2 e^{2}}{h} \sum_{k, l} \partial_{\tau_{\mathrm{E}}} P_{k}^{\mathrm{L}} P_{k}^{\mathrm{R}} P_{k l}\left(\tau_{\mathrm{E}}\right) \int d t P_{l l}(t)
$$

in the absence of a magnetic field. (With a magnetic field, $\delta G_{\mathrm{WL}}=0$.) For the special case of a symmetric double quantum dot this simplifies to

$$
\delta G_{\mathrm{WL}}=-\frac{2 G_{\mathrm{c}}\left(G_{0}+G_{\mathrm{c}}\right)^{2} e^{-\tau_{\mathrm{E}} / \tau_{\mathrm{D}+}}}{\left(G_{0}+2 G_{\mathrm{c}}\right)^{3}} .
$$

Comparing with the expressions for $\delta G_{\mathrm{AA}}$ derived above, we note that both $\delta G_{\mathrm{WL}}$ and $\delta G_{\mathrm{AA}}$ disappear $\propto$ $\exp \left(-\tau_{\mathrm{E}} / \tau_{\mathrm{D}}\right)$ in the limit $\tau_{\mathrm{E}} \gg \tau_{\mathrm{D}}$ at zero temperature. [The fact that $\delta G_{\mathrm{WL}}$ has a single exponential decay rate in Eq. (15) while $\delta G_{\mathrm{AA}}$ has two exponential decay rates is an artifact of the symmetry $G_{1}=G_{2}$.] At finite temperature, $\delta G_{\mathrm{WL}}$ and $\delta G_{\mathrm{AA}}$ have a different dependence on $\tau_{\mathrm{E}}$, since $\delta G_{\mathrm{WL}}$ has no explicit temperature dependence through the thermal time $\hbar / 2 \pi T$.

The exponential dependence of $\delta G_{\mathrm{AA}}$ on $\tau_{\mathrm{E}} / \tau_{\mathrm{D}}$ and $\tau_{\mathrm{E}} T / \hbar$ [Eq. (1)] is the main result of this letter. Although it was derived by explicit calculation of $\delta G_{\mathrm{AA}}$ in a ballistic double quantum dot, we should point out that the origin of the $\tau_{\mathrm{E}}$ dependence of $\delta G_{\mathrm{AA}}$ is in the structure of the classical trajectories contributing to $\delta G_{\mathrm{AA}}$ shown in Fig. 2] not in the details of the semiclassical calculation. It is because of this that we expect that our qualitative conclusions for the $\tau_{\mathrm{E}}$ dependence of $\delta G_{\mathrm{AA}}$ carry over to other geometries, notwithstanding differences in the quantitative evaluation of $\delta G_{\mathrm{AA}}$ in those cases [24].

We thank I. Aleiner for useful discussions. This work was supported by the Cornell Center for Materials research under NSF grant no. DMR 0520404, the Packard Foundation, the Humboldt Foundation, and by the NSF under grant nos. DMR 0334499 and 0705476.

[1] P. W. Anderson, E. Abrahams, and T. V. Ramakrishnan, Phys. Rev. Lett. 43, 718 (1979).
[2] L. P. Gorkov, A. I. Larkin, and D. E. Khmelnitskii, Pis'ma Zh. Eksp. Teor. Fiz. 30, 248 (1979) [JETP Lett. 30, 228 (1979)].

[3] B. L. Altshuler and A. G. Aronov, Solid State Commun. 30, 115 (1979); Zh. Eksp. Teor. Fiz. 77, 2028 (1979) [Sov. Phys. JETP 50, 968 (1979)].

[4] B. L. Altshuler and B. D. Simons, in Mesoscopic Quantum Physics, edited by E. Akkermans, G. Montambaux, J.-L. Pichard, and J. Zinn-Justin (North-Holland, 1995).

[5] C. M. Marcus, A. J. Rimberg, R. M. Westervelt, P. F. Hopkins, and A. C. Gossard, Phys. Rev. Lett. 69, 506 (1992).

[6] H. U. Baranger, R. A. Jalabert, and A. D. Stone, Phys. Rev. Lett. 70, 3876 (1993); Chaos 3, 665 (1993).

[7] In disordered metals, the label "ballistic" is sometimes used for the regime in which the inverse temperature $\hbar / T$ is less than the mean free path $\tau$ for impurity scattering. See, G. Zala, B. N. Narozhny, and I. L. Aleiner, Phys. Rev. B 64, 214204 (2001) for a discussion of the interaction correction in that case.

[8] I. L. Aleiner and A. I. Larkin, Phys. Rev. B 54, 14423 (1996).

[9] A. I. Larkin and Y. N. Ovchinnikov, Zh. Eksp. Teor. Fiz. 55, 2262 (1968) [Sov. Phys. JETP 28, 1200 (1969)].

[10] G. M. Zaslavsky, Phys. Rep. 80, 157 (1981).

[11] I. Adagideli, Phys. Rev. B 68, 233308 (2003); S. Rahav and P. W. Brouwer, Phys. Rev. Lett. 95, 056806 (2005).

[12] A. Altland, P. W. Brouwer, and C. Tian, Phys. Rev. Lett. 99, 036804 (2007).

[13] P. W. Brouwer and I. L. Aleiner, Phys. Rev. Lett. 82, 390 (1999).

[14] P. W. Brouwer, A. Lamacraft, and K. Flensberg, Phys. Rev. Lett. 94, 136801 (2005); Phys. Rev. B 72, 075316 (2005).

[15] I. L. Aleiner, P. W. Brouwer, and L. I. Glazman, Phys. Rep. 358, 309 (2002).

[16] I. L. Aleiner, B. L. Altshuler, and M. E. Gershenson, Waves Random Media 9, 201 (1999).

[17] K. Richter and M. Sieber, Phys. Rev. Lett. 89, 206801 (2002).

[18] M. Gutzwiller, Chaos in Classical and Quantum Mechanics (Springer, New York, 1990).

[19] O. Agam, I. Aleiner, and A. Larkin, Phys. Rev. Lett. 85, 3153 (2000).

[20] R. S. Whitney and P. Jacquod, Phys. Rev. Lett. 96, 206804 (2006).

[21] P. Braun, S. Heusler, S. Müller, and F. Haake, J. Phys. A: Math. Gen. 39, L159 (2006).

[22] P. W. Brouwer, Phys. Rev. B 76, 165313 (2007).

[23] J. N. Kupferschmidt and P. W. Brouwer, unpublished (2007).

[24] This difference is more than the mere adaptation of Eq. (8) to a different classical dynamics, as one can no longer rely on the effectively long-range form of the electronelectron interaction in a general setting. This property of the interaction entered our calculation in two ways: It suppressed the Hartree contribution to $\delta G_{\mathrm{AA}}$ and simplified the trajectory summations leading up to Eq. (8). A theory of the Altshuler-Aronov correction in an arbitrary ballistic conductor would have to deal with both effects. 